\title{
Comportamento ingestivo de cordeiros Santa Inês alimentados com resíduo de cervejaria desidratado
}

[Ingestive behavior of Santa Ines lambs fed rations containing dehydrated brewery residue

\author{
A.G.C.M. Gonçalves ${ }^{1}$, P.G. Pimentel ${ }^{2 *}$, S.P. Gomes ${ }^{3}$, G.R. Moreira ${ }^{4}$, \\ M.S. Feitosa ${ }^{1}$, M.S. Araújo ${ }^{1}$, S.L.S. Soares ${ }^{1}$, S.C. Cardoso ${ }^{5}$, \\ M.R.C. Barroso ${ }^{5}$ \\ ${ }^{1}$ Programa de pós-graduação - Universidade Federal do Ceará - Fortaleza, CE \\ ${ }^{2}$ Universidade Federal do Ceará - Fortaleza, CE \\ ${ }^{3}$ Universidade da Integração Internacional da Lusofonia Afro-Brasileira - Redenção, CE \\ ${ }^{4}$ Universidade Federal Rural de Pernambuco - Recife, PE \\ ${ }^{5}$ Programa de graduação - Universidade Federal do Ceará - Fortaleza, CE
}

\section{RESUMO}

Objetivou-se avaliar o comportamento ingestivo de cordeiros Santa Inês alimentados com resíduo de cervejaria desidratado (RCD). Foram utilizados 35 cordeiros, machos, não castrados, com peso médio

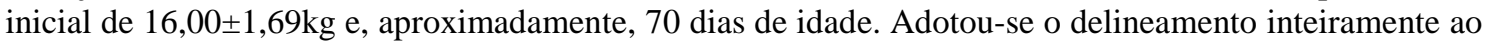
acaso, com cinco tratamentos e sete repetições, consistindo os tratamentos em: $0 ; 20 ; 40 ; 60$ e $80 \%$ de inclusão de RCD na porção concentrada da ração. A inclusão de RCD na ração não influenciou o tempo de alimentação (TAL; h/dia) e a eficiência de alimentação (gFDN/h; P>0,05). O TAL obtido neste estudo apresentou valor médio de 4,90h/dia. Observou-se efeito linear decrescente $(\mathrm{P}<0,05)$ com a inclusão do $\mathrm{RCD}$, para as eficiências de alimentação (gMS/h) e ruminação (gMS/h e gFDN/h). Contudo, efeito linear crescente $(\mathrm{P}<0,05)$ foi constatado para tempo de ruminação e mastigação total, bem como para o número de mastigações merícicas por dia para os animais alimentados com o subproduto. A inclusão de resíduo de cervejaria desidratado influencia o comportamento ingestivo de cordeiros Santa Inês, diminuindo a eficiência de alimentação, quando relacionada ao consumo de matéria seca por hora, e aumentando o tempo de ruminação, podendo ser adicionado em até $20 \%$ na porção concentrada da ração.

Palavras-chave: alimentos alternativos, ovinos, resíduo agroindustrial, ruminantes

\begin{abstract}
The aim of this study was to evaluate the ingestive behavior of Santa Ines lambs fed dehydrated brewer's residue (DBR). Thirty-five male lambs were used, with an initial mean weight of $16.00 \pm 1.69 \mathrm{~kg}$ and, approximately, 70 days of age. A completely randomized design was used, with five treatments and seven replicates, the treatments being: 0; 20; 40; 60 and 80\% of inclusion of DBR in the concentrated portion of the ration. The inclusion of $D B R$ in the ration did not influence feeding time (FT; $h / d a y)$ and feeding efficiency ( $g N D F / h ; P>0.05)$. The FT obtained in this study had an average value of 4.90h/day. There was a decreasing linear effect $(P<0.05)$ for feed efficiency $(g D M / h)$ and rumination efficiency $(g D M / h$ and $g N D F / h)$. However, linear increasing effect $(P<0.05)$ was observed for rumination and total chewing time, as well as for the number of chews per day for animals fed with the byproduct. The inclusion of dehydrated brewer's residue in the concentrate can influence the ingestive behavior of Santa Ines lambs, reducing feed efficiency, when related to the dry matter intake per hour, and increasing the total rumination time in Santa Ines lambs, it can be added up to $20 \%$ in the concentrated portion of the ration.
\end{abstract}

Keywords: agro-industrial waste, alternative feed, ruminant, sheep

Recebido em 21 de setembro de 2017

Aceito em 22 de março de 2019

*Autor para correspondência (corresponding author)

E-mail:pgpimentel@hotmail.com 


\section{INTRODUÇÃO}

Atualmente, a ovinocultura é uma atividade pecuária que se encontra em ascensão, sobretudo na região Nordeste do Brasil. Nesse cenário, destacam-se os sistemas intensivos de produção, com uso de confinamento e suplementação com elevadas proporções de concentrado nas dietas. No entanto, para conseguir competir com a carne de outras espécies, o produtor de carne ovina deve disponibilizar, para o mercado, carne oriunda de animais jovens (cordeiros), manejados adequadamente para obtenção de carcaças de melhor qualidade (Brochier e Carvalho, 2009) e com baixos custos de produção, principalmente relacionados à nutrição do rebanho.

Com a implantação de agroindústrias no Nordeste brasileiro, variados subprodutos são disponibilizados e analisados, a fim de serem aproveitados como alimentos alternativos às culturas tradicionais, contribuindo para a diminuição dos custos com a alimentação animal e para a minimização dos impactos ambientais causados pelo acúmulo de resíduos no ambiente (Pereira et al., 2010). Dentre os subprodutos agroindustriais, destaca-se o resíduo de cervejaria úmido, o qual, sendo produzido em quantidades significativas e não apresentando restrição relacionada à sazonalidade de oferta, pode consistir em ser alternativa viável para alimentação de cordeiros, favorecendo, ainda, a destinação adequada desse resíduo (Brochier e Carvalho, 2008).

A maior limitação do uso do resíduo de cervejaria úmido são os baixos valores de matéria seca (20 a 30\%; West et al., 1994), bem como a dificuldade no transporte e armazenamento, sendo a sua desidratação uma alternativa para viabilizar seu uso. Entretanto, a ausência de dados na literatura sobre a composição físico-química de alimentos ditos alternativos indica a falta de caracterização e de informações nutricionais que permitam a sua recomendação de forma mais abrangente. Assim, conhecer fatores como a composição química e a digestibilidade do alimento, bem como os processos fisiológicos e metabólicos envolvidos, é fundamental para estabelecer seu valor nutricional e critérios para sua inclusão nas rações (Pereira et al., 2010).
Nesse contexto, os estudos sobre comportamento ingestivo possibilitam avaliar as características dos alimentos, a motilidade do rúmen-retículo e o ambiente, sendo considerados ferramentas de ajuste no manejo alimentar para obtenção do melhor desempenho produtivo dos animais (Macedo Júnior et al., 2009). Objetivou-se, com o presente estudo, avaliar o comportamento ingestivo de cordeiros Santa Inês alimentados com resíduo de cervejaria desidratado na porção concentrada da ração.

\section{MATERIAL E MÉTODOS}

O experimento foi conduzido no Setor de Digestibilidade, pertencente ao Departamento de Zootecnia da Universidade Federal do Ceará, em Fortaleza, Ceará, de acordo com os princípios éticos na experimentação animal (Protocolo 36/2015) determinados pelo Conselho de Ética em Experimentação Animal da referida instituição. O resíduo de cervejaria foi adquirido na forma úmida de uma indústria cervejeira, localizada na Região Metropolitana de Fortaleza e, posteriormente, desidratado ao sol. Para realizar a desidratação, o resíduo foi distribuído em lonas plásticas e exposto ao sol durante oito dias, no horário de oito às 17 horas, sendo revolvido a cada 60 minutos e recolhido ao final da tarde. Após a secagem, o resíduo de cervejaria desidratado (RCD) foi pesado, acondicionado em sacos plásticos e, posteriormente, incluído na proporção de $0 ; 20 ; 40 ; 60$ e $80 \%$ da porção concentrada das rações avaliadas.

Foram utilizados 35 cordeiros Santa Inês, com, aproximadamente, 70 dias de idade e com peso corporal médio de $16,00 \pm 1,69 \mathrm{~kg}$, os quais foram alojados em baias individuais de confinamento, providas de comedouros e bebedouros, com água permanentemente à disposição. Foi estabelecido período de 21 dias para adaptação às rações experimentais e às instalações, e, após esse período, os animais foram confinados por 74 dias. O delineamento experimental utilizado foi o inteiramente ao acaso, com cinco tratamentos e sete repetições. Os tratamentos experimentais consistiram em cinco níveis de inclusão do RCD $(0 ; 20 ; 40 ; 60$ e $80 \%)$ na porção concentrada da ração. As rações experimentais foram formuladas de acordo com as recomendações do NRC (Nutrient..., 2007) para ganho médio diário de $200 \mathrm{~g}$, com relação volumoso:concentrado de 60:40. O volumoso foi constituído por feno de 
Tifton 85 (Cynodon dactylon), e o concentrado à base de farelo de soja, milho em grão moído, resíduo de cervejaria desidratado nas proporções avaliadas, fosfato bicálcico e mistura mineral (Tab. 1 e 2). O fornecimento das rações experimentais foi realizado duas vezes ao dia (oito e 16 horas), e a quantidade de alimento oferecida ajustada diariamente, com base no consumo observado no dia anterior, permitindose sobras de $10 \%$, caracterizando consumo ad libitum.

Tabela 1. Composição químico-bromatológica (\%) dos ingredientes e das rações concentradas experimentais contendo resíduo de cervejaria desidratado (RCD)

\begin{tabular}{|c|c|c|c|c|c|c|c|c|c|}
\hline \multirow[b]{2}{*}{ Nutriente } & \multicolumn{4}{|c|}{ Ingredientes } & \multicolumn{5}{|c|}{ Resíduo de cervejaria desidratado $(\%)^{1}$} \\
\hline & $\begin{array}{l}\text { Feno de } \\
\text { Tifton } 85\end{array}$ & $\begin{array}{l}\text { Milho } \\
\text { grão } \\
\text { moído }\end{array}$ & $\begin{array}{l}\text { Farelo } \\
\text { de soja }\end{array}$ & RCD & 0 & 20 & 40 & 60 & 80 \\
\hline $\mathrm{MS}^{2}$ & 92,88 & 93,40 & 94,48 & 93,79 & 93,09 & 93,10 & 95,61 & 93,03 & 95,87 \\
\hline $\mathrm{PB}^{3}$ & 7,47 & 8,77 & 49,36 & 28,87 & 21,32 & 22,96 & 22,35 & 23,05 & 24,80 \\
\hline $\mathrm{MM}^{4}$ & 5,80 & 1,52 & 7,20 & 3,43 & 6,01 & 5,94 & 5,40 & 5,44 & 5,01 \\
\hline $\mathrm{EE}^{5}$ & 2,20 & 4,90 & 2,05 & 7,87 & 3,72 & 4,51 & 5,35 & 5,64 & 6,83 \\
\hline $\mathrm{FDN}^{6}$ & 82,64 & 16,94 & 13,07 & 56,21 & 13,47 & 24,45 & 34,24 & 45,37 & 49,07 \\
\hline $\mathrm{FDNcp}^{7}$ & 77,61 & 15,78 & 7,29 & 42,76 & 11,51 & 20,19 & 28,08 & 36,60 & 38,81 \\
\hline $\mathrm{FDA}^{8}$ & 44,42 & 6,78 & 7,82 & 15,53 & 9,59 & 20,64 & 23,08 & 25,72 & 29,27 \\
\hline $\mathrm{CHOT}^{9}$ & 84,54 & 84,80 & 41,39 & 59,83 & 68,94 & 66,59 & 66,91 & 65,87 & 63,36 \\
\hline $\mathrm{CNF}^{10}$ & 6,93 & 69,02 & 34,10 & 19,31 & 57,44 & 46,40 & 38,83 & 29,28 & 24,55 \\
\hline
\end{tabular}

${ }^{1}$ Níveis de inclusão de RCD na porção concentrada da ração; ${ }^{2}$ matéria seca; ${ }^{3}$ proteína bruta; ${ }^{4}$ matéria mineral; ${ }^{5}$ extrato etéreo; ${ }^{6}$ fibra em detergente neutro; ${ }^{7}$ fibra em detergente neutro corrigido para cinzas e proteína; ${ }^{8}$ fibra em detergente ácido; ${ }^{9}$ carboidratos totais; ${ }^{10}$ carboidratos não fibrosos.

Tabela 2. Composição percentual e químico-bromatológica das rações experimentais contendo resíduo de cervejaria desidratado (RCD)

\begin{tabular}{|c|c|c|c|c|c|}
\hline \multirow{2}{*}{ Ingredientes (\%MN) } & \multicolumn{5}{|c|}{ Resíduo de cervejaria desidratado (\%) } \\
\hline & 0 & 20 & 40 & 60 & 80 \\
\hline Feno de Tifton 85 & 60 & 60 & 60 & 60 & 60 \\
\hline Concentrado $^{1}$ & 40 & 40 & 40 & 40 & 40 \\
\hline Milho grão moído & 27,60 & 22,80 & 18,20 & 13,44 & 6,80 \\
\hline Farelo de soja & 11,20 & 8,00 & 4,60 & 1,36 & 0,00 \\
\hline $\mathrm{RCD}$ & 0,00 & 8,00 & 16,00 & 24,00 & 32,00 \\
\hline Fosfato bicálcico ${ }^{2}$ & 0,20 & 0,20 & 0,20 & 0,20 & 0,20 \\
\hline Mistura mineral $^{3}$ & 1,00 & 1,00 & 1,00 & 1,00 & 1,00 \\
\hline \multicolumn{6}{|c|}{ Composição química (\%MS) } \\
\hline $\mathrm{MS}^{4}$ & 93,09 & 93,16 & 93,16 & 93,15 & 93,17 \\
\hline $\mathrm{PB}^{5}$ & 13,58 & 14,03 & 13,89 & 14,28 & 14,01 \\
\hline $\mathrm{MM}^{6}$ & 5,94 & 5,85 & 5,63 & 5,69 & 5,46 \\
\hline $\mathrm{EE}^{7}$ & 3,05 & 3,40 & 3,88 & 4,08 & 4,24 \\
\hline $\mathrm{FDN}^{8}$ & 50,17 & 55,49 & 59,92 & 64,81 & 67,02 \\
\hline FDNcp $^{9}$ & 51,74 & 54,17 & 56,62 & 59,05 & 61,32 \\
\hline $\mathrm{FDA}^{10}$ & 28,09 & 33,80 & 34,49 & 35,46 & 38,24 \\
\hline Lignina & 5,01 & 8,31 & 9,40 & 10,81 & 11,89 \\
\hline $\mathrm{CHOT}^{11}$ & 77,36 & 76,63 & 76,22 & 75,68 & 76,11 \\
\hline $\mathrm{CNF}^{12}$ & 27,03 & 24,17 & 21,38 & 18,54 & 15,03 \\
\hline $\mathrm{NDT}^{13}$ & 51,81 & 49,14 & 49,14 & 53,32 & 53,72 \\
\hline NDT:PB ${ }^{14}$ & 3,82 & 3,43 & 3,54 & 3,73 & 3,83 \\
\hline \multicolumn{6}{|c|}{$\begin{array}{l}{ }^{1} \text { Composição centesimal em relação à ração total; }{ }^{2} \text { composição: Ca } 24 \% \text {; P } 18 \% ;{ }^{3} \text { composição: Ca } 7,5 \% ; \text { P } 3 \% \text {; Fe } \\
16.500 \mathrm{ppm} \text {; Mn 9.750ppm; Zn 35.000ppm; I 1.000ppm; Se } 225 \mathrm{ppm} \text {; Co } 1.000 \mathrm{ppm} ;{ }^{4} \text { matéria seca; }{ }^{5} \text { proteína bruta; } \\
{ }^{6} \text { matéria mineral; }{ }^{7} \text { extrato etéreo; }{ }^{8} \text { fibra em detergente neutro; }{ }^{9} \text { fibra em detergente neutro corrigido para cinzas e } \\
\text { proteína; }{ }^{10} \text { fibra em detergente ácido; }{ }^{11} \text { carboidratos totais; }{ }^{12} \text { carboidratos não fibrosos; }{ }^{13} \text { nutrientes digestíveis totais; }\end{array}$} \\
\hline
\end{tabular}


Amostras das sobras, dos ingredientes, do feno e dos concentrados foram armazenadas congeladas $\left(-10^{\circ} \mathrm{C}\right)$ para formação posterior de amostra composta por animal por tratamento. A determinação do teor de matéria seca (MS), proteína bruta $(\mathrm{PB})$, matéria mineral $(\mathrm{MM}) \mathrm{e}$ extrato etéreo (EE) foi realizada segundo métodos da AOAC (Official..., 1990). A fibra em detergente neutro (FDN), a fibra em detergente neutro corrigida para cinzas e proteína (FDNcp), a fibra em detergente ácido (FDA) e a lignina foram realizadas de acordo com o método descrito por Van Soest et al. (1991). Os teores de carboidratos totais (CHOT) foram obtidos segundo Sniffen et al. (1992): \%CHOT = 100 $(\% \mathrm{~PB}+\% \mathrm{EE}+\% \mathrm{MM})$, e os carboidratos não fibrosos (CNF) conforme a equação proposta por Weiss (1999): \%CNF $=100-(\%$ FDNcp $+\%$ PB $+\% \mathrm{EE}+\% \mathrm{MM})$. Os nutrientes digestíveis totais (NDT) foram calculados de acordo com a seguinte fórmula (Weiss, 1999): \%NDT $=\mathrm{PBd}+$ CNFd + FDNcpd + EEd x 2,25; em que: PBd, CNFd, FNDcpd e EEd correspondem aos respectivos nutrientes digestíveis.

Para mensuração do comportamento ingestivo, os animais foram submetidos à observação visual no $40^{\circ}$ e $41^{\circ}$ dias experimentais. No primeiro dia, foi realizada observação visual, em intervalos de cinco minutos, durante 24 horas, para determinação do tempo despendido com alimentação, ruminação e ócio, conforme metodologia proposta por Johnson e Combs (1991). No segundo dia, os animais foram observados durante três períodos de duas horas (oito às $10 ; 14$ às 16 e 18 às 20 horas), sendo coletadas informações para se estimar o número de mastigações merícicas por bolo ruminal e o tempo despendido com mastigação merícica por bolo ruminal, utilizando-se cronômetro digital. O galpão experimental foi mantido sob iluminação artificial à noite, durante todo o período experimental.

As variáveis relacionadas ao comportamento ingestivo foram obtidas pelas equações: EAL = $\mathrm{CMS} / \mathrm{TAL} ; \mathrm{EAL}=\mathrm{CFDN} / \mathrm{TAL} ; \mathrm{ERU}=$ $\mathrm{CMS} / \mathrm{TRU}$; ERU $=\mathrm{CFDN} / \mathrm{TRU}$; TMT $=\mathrm{TAL}+$ TRU; $\mathrm{NBR}=$ TRU/MMtb; $\mathrm{MMnd}=$ NBR*MMnb, em que: EAL (gMS/h, gFDN/h) corresponde à eficiência de alimentação; CMS (gMS/dia), ao consumo de MS; TAL (h/dia), ao tempo de alimentação; CFDN (gFDN/dia), ao consumo de FDN; ERU (gMS/h, gFDN/h), à eficiência de ruminação; TRU (h/dia), ao tempo de ruminação; TMT (h/dia), ao tempo de mastigação total; NBR ( ${ }^{\circ} /$ dia), ao número de bolos ruminais; MMtb (seg/bolo) corresponde ao tempo de mastigação merícica por bolo ruminal; MMnd ( $\mathrm{n}^{\circ} /$ bolo), ao número de mastigações por dia; e MMnb ( $\mathrm{n}^{\circ} /$ bolo), ao número de mastigações merícicas por bolo ruminal (Polli et al., 1996).

As variáveis foram submetidas, previamente, à verificação de normalidade e homocedasticidade. Posteriormente, foi realizada análise de variância e, sendo os tratamentos quantitativos, estes foram analisados por meio do desdobramento dos tratamentos em regressão linear, quadrática e desvio por meio do teste $\mathrm{F}(\mathrm{P}<0,05)$, utilizandose o programa computacional Sisvar 5.3 Build 77 (Ferreira, 2011).

\section{RESULTADOS E DISCUSSÃO}

A inclusão de RCD na ração não influenciou (P>0,05) o tempo de alimentação (TAL) e a eficiência de alimentação (EAL) relacionada ao consumo de FDN por hora (Tab. 3), os quais apresentaram valor médio de 4,90h/dia e $112,39 \mathrm{gFDN} / \mathrm{h}$, respectivamente. Possivelmente, a ausência de diferença para o consumo de FDN (valor médio de 530,31g/dia) e para o TAL $(\mathrm{h} / \mathrm{dia} ; \mathrm{P}>0,05)$ resultou em semelhante $(\mathrm{P}>0,05)$ eficiência de alimentação, quando expressa em gFDN/h.

Mendonça (2012), corroborando o presente estudo, não observou efeito significativo ( $\mathrm{P}>0,05)$ no TAL $(3,40 \mathrm{~h} /$ dia $)$ ao avaliar quatro níveis de inclusão de resíduo de cervejaria úmido na ração de cabras leiteiras $(0 ; 15 ; 20$ e $25 \%)$ em substituição ao farelo de soja no concentrado. No entanto, Frasson et al. (2016), ao fornecerem resíduo de cervejaria úmido na ração para cordeiros $(0 ; 33,5 ; 66,5$ e $100 \%$ do subproduto), verificaram comportamento linear decrescente para o TAL $(3,42 ; 3,39 ; 2,94$ e $2,82 \mathrm{~h} / \mathrm{dia}$, respectivamente). 
Comportamento ingestivo de...

Tabela 3. Comportamento ingestivo de cordeiros Santa Inês alimentados com resíduo de cervejaria desidratado

\begin{tabular}{|c|c|c|c|c|c|c|c|}
\hline \multirow{2}{*}{ Variáveis } & \multicolumn{5}{|c|}{ Resíduo de cervejaria desidratado (\%) } & \multirow{2}{*}{ EPM $^{1}$} & \multirow{2}{*}{ Valor-P* } \\
\hline & 0 & 20 & 40 & 60 & 80 & & \\
\hline TAL $(\mathrm{h} / \mathrm{dia})^{2}$ & 4,75 & 4,46 & 4,55 & 5,57 & 5,19 & - & 0,0535 \\
\hline EAL $(\mathrm{gMS} / \mathrm{h})^{3}$ & 224,16 & 226,51 & 202,66 & 149,86 & 156,48 & 8,13 & $<0,0001$ \\
\hline EAL $(\mathrm{gFDN} / \mathrm{h})^{4}$ & 112,80 & 125,69 & 121,52 & 97,26 & 104,68 & - & 0,0960 \\
\hline TRU (h/dia) $)^{5}$ & 8,55 & 8,23 & 10,09 & 9,61 & 10,26 & 0,26 & 0,0054 \\
\hline ERU $(\mathrm{gMS} / \mathrm{h})^{6}$ & 126,61 & 120,56 & 91,86 & 84,03 & 78,59 & 4,41 & $<0,0001$ \\
\hline ERU $(\mathrm{gFDN} / \mathrm{h})^{7}$ & 63,65 & 66,91 & 55,02 & 54,49 & 52,64 & 1,83 & 0,0062 \\
\hline TMT $(\mathrm{h} / \mathrm{dia})^{8}$ & 13,30 & 12,69 & 14,64 & 15,18 & 15,45 & 0,30 & 0,0006 \\
\hline Ócio $(\mathrm{h} / \mathrm{dia})^{9}$ & 10,36 & 11,12 & 9,07 & 8,52 & 8,40 & 0,31 & 0,0014 \\
\hline
\end{tabular}

${ }^{1}$ Erro-padrão da média; ${ }^{2} \tilde{\mathrm{Y}}=4,90{ }^{\mathrm{NS}} ;{ }^{3} \hat{\mathrm{Y}}=234,33-1,06 \mathrm{X}\left(\mathrm{R}^{2}=0,84\right) ;{ }^{4} \tilde{\mathrm{Y}}=112,39^{\mathrm{NS}} ;{ }^{5} \hat{\mathrm{Y}}=8,39+0,02 \mathrm{X}\left(\mathrm{R}^{2}=0,69\right)$; ${ }^{6} \hat{\mathrm{Y}}=116,685-0,66 \mathrm{X}\left(\mathrm{R}^{2}=0,92\right) ;{ }^{7} \hat{\mathrm{Y}}=65,43-0,17 \mathrm{X}\left(\mathrm{R}^{2}=0,74\right) ;{ }^{8} \hat{\mathrm{Y}}=12,89+0,03 \mathrm{X}\left(\mathrm{R}^{2}=0,80\right) ;{ }^{9} \hat{\mathrm{Y}}=10,80-0,03 \mathrm{X}$ $\left(\mathrm{R}^{2}=0,74\right)$; TAL= tempo de alimentação; $\mathrm{EAL}=$ eficiência de alimentação; TRU= tempo de ruminação; ERU= eficiência de ruminação; TMT= tempo de mastigação total. NS= Não significativo. *Significativo a $5 \%$ de probabilidade.

Observou-se efeito linear decrescente, com a inclusão do RCD na ração, para a EAL, em $\mathrm{gMS} / \mathrm{h}$, e para a eficiência de ruminação (ERU), em $\mathrm{gMS} / \mathrm{h}$ e $\mathrm{gFDN} / \mathrm{h}$, podendo-se inferir que o fator que contribuiu para esse comportamento foi o consumo de MS, o qual reduziu linearmente com a inclusão do RCD à ração $(\hat{Y}=1016,49$ $\left.2,92 \mathrm{X} ; \mathrm{R}^{2}=0,96\right)$. Estudos conduzidos por Dulphy et al. (1980) mostraram que a eficiência de ruminação, em gramas por hora, pode ser reduzida para dietas com alta fibra, em virtude da maior dificuldade em reduzir o tamanho das partículas oriundas de materiais fibrosos.

O tempo de ruminação (TRU) apresentou efeito linear crescente $(\mathrm{P}<0,05)$ com a inclusão do RCD na ração, corroborando os estudos de Macedo Júnior et al. (2009), que descreveram que o tempo gasto com ruminação aumenta linearmente com a elevação no teor de FDN das rações. Tal observação se deve ao fato de que rações com alto conteúdo de FDN necessitam de maior tempo para processamento da fibra, uma vez que, sendo esta a fração menos digestível dos alimentos, precisará de maior atividade mastigatória para redução do tamanho das partículas e maior tempo para digestão nos préestômagos. Por outro lado, o maior tempo de ruminação promove maior produção de saliva, consequentemente, aumentando o fluxo de tampões para os pré-estômagos, o que promove a melhora da saúde do ambiente ruminal e a digestão da fibra (Dulphy et al., 1980).
No presente estudo, verificou-se que, a cada $1 \%$ de RCD incluído na ração, ocorreu aumento de 0,02h/dia no TRU (Tab. 3), possivelmente, em virtude do elevado conteúdo de parede celular (FDN e FDA) das rações com inclusão do RCD (Tab. 2). Diferentemente do observado neste estudo, Frasson et al. (2016) constataram comportamento linear decrescente para a referida atividade (TRU médios de 7,61;7,42; 7,61 e $6,14 \mathrm{~h} / \mathrm{dia}$ ) ao fornecerem resíduo de cervejaria úmido para cordeiros.

Com relação ao tempo de ócio, observou-se redução linear $(\mathrm{P}<0,05)$ com a adição do RCD às rações, em que, a cada $1 \%$ de inclusão do RCD, os cordeiros diminuíam essa atividade em 0,03h/dia. Da mesma forma, Carvalho et al. (2006), ao avaliarem o comportamento ingestivo de cabras consumindo níveis crescentes de FDN $(20 ; 27 ; 34 ; 41$ e $48 \%)$ provenientes de forragem, observaram diminuição do tempo de ócio com a elevação dos níveis da FDN na ração. Contudo, a redução linear no tempo despendido com as atividades de alimentação e ruminação e, consequentemente, no tempo de mastigação total, resultou em aumento linear do tempo de ócio, em estudo conduzido por Frasson et al. (2016), em que se utilizou níveis crescentes de resíduo de cervejaria úmido na alimentação de cordeiros.

A constatação de que houve aumento no tempo destinado à ruminação e redução do tempo de 
descanso dos animais (ócio) é relevante, pois implica em aumento de atividade física, que despende energia (Macedo et al., 2007), inferindo-se, então, que o aumento dos níveis de inclusão de RCD na ração pode determinar aumento nas exigências de energia de mantença, podendo contribuir para reduzir o desempenho do animal. No entanto, como citado anteriormente, maior tempo de ruminação é importante para a manutenção de um ambiente ruminal saudável.

O tempo de mastigação total (Tab. 3) e o número de mastigações merícicas por dia (MMnd; Tab. 4) apresentaram efeito linear crescente $(\mathrm{P}<0,05)$ com a inclusão de RCD nas rações, podendo-se inferir que, possivelmente, o aumento da necessidade de mastigação está relacionada à quantidade de material indigestível ou pouco digestível presente no RCD, bem como à resistência do subproduto à redução no tamanho das partículas.
Mendes et al. (2010), ao avaliarem duas fontes de FDN (bagaço de cana in natura ou casca de soja), dois teores de FDN (14 e 18\%) e uma ração controle $(100 \%$ de concentrado), observaram que as rações com fonte adicional de fibra influenciaram o tempo de ingestão e ruminação em cordeiros Santa Inês e que, dependendo da fonte de FDN fornecida, o tempo de mastigação apresentou respostas antagônicas. Os autores afirmaram que a mastigação estimula a secreção de saliva e os tamponantes produzidos auxiliam na neutralização dos ácidos orgânicos produzidos durante o processo de fermentação ruminal. A inclusão de $\mathrm{RCD}$ na ração não influenciou o número de bolos ruminais (NBR), o número de mastigações merícicas por bolo ruminal (MMnb) e o tempo de mastigação merícica por bolo ruminal (MMtb; P>0,05; Tab. 4).

Tabela 4. Comportamento ingestivo de cordeiros Santa Inês alimentados com resíduo de cervejaria desidratado

\begin{tabular}{|c|c|c|c|c|c|c|c|}
\hline \multirow{2}{*}{ Variáveis } & \multicolumn{5}{|c|}{ Resíduo de cervejaria desidratado (\%) } & \multirow{2}{*}{$\mathrm{EPM}^{1}$} & \multirow{2}{*}{ Valor-P* } \\
\hline & 0 & 20 & 40 & 60 & 80 & & \\
\hline $\operatorname{NBR}\left(n^{\circ} / \text { dia }\right)^{2}$ & 763,80 & 669,62 & 812,40 & 772,53 & 827,15 & - & 0,1059 \\
\hline MMnd (n⿳/dia) ${ }^{3}$ & 45929 & 41805 & 50175 & 59997 & 53644 & 1321 & 0,0062 \\
\hline MMnb (n\%/bolo) ${ }^{4}$ & 60,99 & 63,56 & 62,81 & 66,48 & 64,81 & - & 0,3555 \\
\hline MMtb (seg/bolo) $)^{5}$ & 40,63 & 45,13 & 45,39 & 45,13 & 44,65 & - & 0,3064 \\
\hline
\end{tabular}

${ }^{1}$ Erro-padrão da média; ${ }^{2} \tilde{Y}=769,10^{\mathrm{NS}} ;{ }^{3} \hat{\mathrm{Y}}=43586+123,11 \mathrm{X}\left(\mathrm{R}^{2}=0,70\right) ;{ }^{4} \tilde{\mathrm{Y}}=63,73^{\mathrm{NS}} ;{ }^{5} \tilde{\mathrm{Y}}=44,19^{\mathrm{NS}} ; \mathrm{NBR}=$ número de bolos ruminais; $\mathrm{MMnd}=$ número de mastigações merícicas por dia; $\mathrm{MMnb}=$ número de mastigações merícicas por bolo ruminal; $\mathrm{MMtb}=$ tempo de mastigações merícicas por bolo ruminal; $\mathrm{NS}=$ não significativo. *Significativo a $5 \%$ de probabilidade.

Camilo et al. (2012) observaram que a redução linear no fornecimento de fibra para cordeiros Morada Nova influenciou $(\mathrm{P}<0,05) \quad \mathrm{o}$ comportamento ingestivo dos animais, com exceção da eficiência de ruminação, NBR, MMnd e MMnb (31,958gFDN/h, 634,4n\% dia, $36610 \mathrm{n} /$ dia e $57,61 \mathrm{n}^{\circ} /$ bolo, respectivamente). Os autores reportaram que, possivelmente, a redução gradual no conteúdo de parede celular da ração, com concomitante aumento na concentração de amido, resultou em redução no tempo de mastigação total $(14,61 ; 13,58 ; 12,00 ; 9,98 \mathrm{e}$ $7,91 \mathrm{~h} /$ dia para consumos de FDN de 237,03; 278,$87 ; 265,89 ; \quad 238,72$ e $187,99 \mathrm{~g} / \mathrm{dia}$, respectivamente). Alterações nos tempos despendidos nas atividades de alimentação, ruminação e ócio têm sido frequentemente observadas em pesquisas nas quais as rações experimentais apresentam variações nos teores de fibra (Hübner et al., 2008; Missio et al., 2010), como foi constatado neste estudo, com a adição de RCD no concentrado de cordeiros.

\section{CONCLUSÃO}

A inclusão de resíduo de cervejaria desidratado influencia o comportamento ingestivo de cordeiros Santa Inês, diminuindo a eficiência de alimentação, quando relacionada ao consumo de matéria seca por hora, e aumentando o tempo de ruminação, podendo ser esse resíduo adicionado em até $20 \%$ na porção concentrada da ração. 


\section{AGRADECIMENTOS}

Ao Conselho Nacional de Desenvolvimento Científico e Tecnológico (CNPq), pelo suporte financeiro à pesquisa (Proc. 474447/2013-8).

\section{REFERÊNCIAS}

BROCHIER, M.A.; CARVALHO, S. Consumo, ganho de peso e análise econômica da terminação de cordeiros em confinamento com dietas contendo diferentes proporções de resíduo úmido de cervejaria. Arq. Bras. Med. Vet. Zootec., v.60, p.1205-1212, 2008.

BROCHIER, M.A.; CARVALHO, S. Effect of different proportions of wet brewery residue on the carcass characteristics of feedlot finished lambs. Arq. Bras. Med. Vet. Zootec., v.61, p.190195, 2009.

CAMILO, D.A.; PEREIRA, E.S.; PIMENTEL, P.G. et al. Intake and feeding behaviour of Morada Nova lambs fed diferente energy levels. Ital. J. Anim. Sci., v.11, p.13-19, 2012.

CARVALHO, S.; RODRIGUES, M.T.; BRANCO, R.H. et al. Comportamento ingestivo de cabras Alpinas em lactação alimentadas com dietas contendo diferentes níveis de fibra em detergente neutro proveniente da forragem. Rev. Bras. Zootec., v.35, p.562-568, 2006.

DULPHY, J.P.; REMOND, B.; THERIEZ, M. Ingestive behaviour and related activities in ruminants. In: RUCKEBUSH, Y., THIVEND, P. (Eds.). Digestive physiology and metabolism in ruminants. Lancaster: MTP, 1980. p.103-122.

FERREIRA, D.F. Sisvar: a computer statistical analysis system. Ciênc. Agrotec., v.35, p.10391042, 2011.

FRASSON, M.F.; CARVALHO, S.; PIRES, C.C. et al. Comportamento ingestivo e produtivo de cordeiros alimentados com resíduo úmido de cervejaria em substituição a silagem de sorgo. Arch. Zootec., v.65, p.183-190, 2016.

HÜBNER, G.H.; PIRES, C.C.; GALVANI, D.B. et al. Comportamento ingestivo de ovelhas em lactação alimentadas com dietas contendo diferentes níveis de fibra em detergente neutro. Ciênc. Rural, v.38, p.1078-1084, 2008.
JOHNSON, T.R.; COMBS, D.K. Effects of prepartum diet, inert rumen bulk, and dietary polythylene glicol on dry matter intake of lactating dairy cows. J. Dairy Sci., v.74, p.933944, 1991.

MACEDO JUNIOR, G.L.; FRANÇA, P.M.; ASSIS, T.R.V. et al. Level of fiber on feeding of Santa Ines pregnant ewes. Arq. Bras. Med. Vet. Zootec., v.61, p.196-202, 2009.

MACEDO, C.A.B.; MIZUBUTI, I.Y.; MOREIRA, B.F. et al. Comportamento ingestivo de ovinos recebendo dietas com diferentes níveis de bagaço de laranja em substituição à silagem de sorgo na ração. Rev. Bras. Zootec., v.36, p.1910-1916, 2007.

MENDES, C.Q.; TURINO, V.F.; SUSIN, I. et al. Comportamento ingestivo de cordeiros e digestibilidade dos nutrientes de dietas contendo alta proporção de concentrado e diferentes fontes de fibra em detergente neutro. Rev. Bras. Zootec., v.39, p.594-600, 2010.

MENDONÇA, L.M. Utilização do resíduo úmido de cervejaria na alimentação de cabras anglo-nubiana em final de lactação. 2012. 66f. Dissertação (Mestrado em Agroecossistemas) Universidade Federal de Sergipe, Aracaju, SE.

MISSIO, R.L.; BRONDANI, I.L.; ALVES FILHO, D.C. et al. Comportamento ingestivo de tourinhos terminados em confinamento, alimentados com diferentes níveis de concentrado na dieta. Rev. Bras. Zootec., v.39, p.1571-1578, 2010.

NUTRIENT requirements of small ruminants. 7.ed. Washington: National Academic Press, 2007. 408p.

OFFICIAL methods of analysis. 15.ed. Virginia: AOAC, 1990. 1117p.

PEREIRA, E.S.; PIMENTEL, P.G.; DUARTE, L.S. et al. Determinação das frações proteicas e de carboidratos e estimativa do valor energético de forrageiras e subprodutos da agroindústria produzidos no Nordeste Brasileiro. Semin. Ciênc. Agrár., v.31, p.1079-1094, 2010.

POLLI, V.A.; RESTLE, J.; SENNA, D.B. et al. Aspectos relativos à ruminação de bovinos $\mathrm{e}$ bubalinos em regime de confinamento. Rev. Bras. Zootec., v.25, p.987-993, 1996. 


\section{Gonçalves et al.}

SNIFFEN, C.J.; O'CONNOR， D.J.; VAN SOEST, P.T. et al. A net carbohydrate and protein system for evaluating cattle diets: carbohydrate and protein availability. J. Anim. Sci., v.70, p.3562-3577, 1992.

VAN SOEST, P.J.; ROBERTSON, J.B.; LEWIS, B.A. Methods for dietary fiber, neutral-detergent fiber and nonstarch polysaccharides in relation to animal nutrition. J. Dairy Sci., v.74, p.35833597, 1991.
WEISS, W.P. Energy prediction equations for ruminant feeds. In: CORNELL NUTRUTION CONFERENCE FEED MANUFACTURES, 61., 1999, Ithaca. Proceedings... Ithaca: Cornell University, 1999. p.176-185.

WEST, J.W.; ELY, L.O.; MARTIN, S.A. Wet brewers' grains for lactating dairy cows during hot, humid weather. J. Dairy Sci., v.77, p.196204, 1994. 